\title{
Highlights of AAPS in Vitro Release and Dissolution Testing Focus Group Meeting
}

\author{
Vivian A. Gray ${ }^{1}$ and Diane Worrell ${ }^{2}$ \\ ${ }^{1}$ Corresponding author, V. A. Gray Consulting, Inc, Hockessin, DE \\ ${ }^{2}$ Manager, Analytics-Dissolution, Quintiles, Inc., Kansas City, MO
}

email:vagray@rcn.com

On April 29,2005, the second face to face meeting of the AAPS in Vitro Release and Dissolution Testing Focus Group took place at the

Pfizer site in Groton, CT. There were about 35 attendees including those that called in.

There were brief presentations by Tahseen Mirza, Vivian Gray, Bryan Crist, Kailas Thakker, Alger Salt, Diane Worrell, Terry Wilke, and Fan Jin.

The slides are on the website http://www.aapspharmaceutica.com/inside/focus_groups/InVitro/index.asp.

Rather than try to record all the discussions at the meeting, what will follow are the hot topics and questions that will be either pursued as a paper or folded into the workshop planned for 2006 or both.

1. What is the current thinking from industry and FDA about having to have $100 \%$ released? Is $80 \%$ okay, especially with novel and modified dosage forms?

2. The use of hydroalcoholic medium. Used to be forbidden but is that changing? What is FDA thinking about this? Topicals sometime use hydroalcoholic medium in the diffusion cells. Is it permissible with an IVIVC?

3. How are people handling evaporation control with the long testing time procedures?

4. What about accelerated testing? Increasing bath temperature?

5. How to handle method development with very limited or expensive number of dosage units? What about setting specs in this situation?

6. New Transdermal dosage forms are under development and need some focus as far as equipment and methods.

7. Would be useful to have a list and preparation of other simulated fluids, i. e, saliva, intramuscular, saline, colon...

8. Need to distinguish between local and systemic absorption of topicals.

9. Fiber optics is viable analytical tool and being examined by many labs. Labs still need to do in depth study of which type of set up is best for their products. Validation against conventional UV or HPLC still needed? What about background corrections and second derivative corrections? Calibration of optical cells can take time?

10. Automation-when do you validate against manual method, if ever? Can it be the primary method?

11. Automation and hydrodynamic effects-are they being correctly evaluated?

12. When do you file the automated method?

13. Is $\mathrm{f} 2$ the method of choice for validation criteria or criteria in proposed USP < 1092> Dissolution Procedure: Development and Validation General Chapter? Are these criteria too lenient?

14. Validation- in vessel versus bench top flasks? How far to take robustness? Criteria for placebo interference 2\%. 3\%, higher?? Is pH change measurement during test important? Validate the profile points or single point? Is the $y$-intercept in linearity important? Should you validate down to zero?

15. Validation-different requirements depending on phase?

16. Filter validation-still underrated?

17. Is HPLC or UV the preferred analytical method?

18. HPLC autosamplers cooled, ambient?

19. Deaeration in the robustness validation - when do you do it? How do you do it?

20. How do you measure deaeration?

21. More mathematical modeling should be used?

22. Suspensions - seems to be a big questions about how you sample the dosage unit, how do you shake. ...many questions around suspension methodology.

23. Method development - is it being done to include challenges to the ability of the test to pick up change? Is it being developed to pick up the critical manufacturing variables?

24. Should you try to file any data showing that the method is discriminating? Should you file an IVIVR?

25. What do you do about coning?

26. What about surfactants? An exhaustive list of surfactants would be useful. What are strengths and weaknesses of each?

27. Using a specific surfactant should have in depth scientific reasoning. More needs to be known about the chemistry of the specific surfactants with the specific formulation and drugs.

28. FDA initiatives -PAT, QBD (Quality by design) and latest initiative to replace calibrator tablets with enhanced calibration and in house calibrators. How is the focus group addressing this initiative?

29. Need to get workshop program going soon. Should include FDA, USP, industry and other speakers on PAT on how these initiatives effect dissolution.

The URL address for the AAPS IVDTFG is http://www. aapspharmaceutica.com/inside/focus_groups/InVitro/index.asp In addition to presentations, the website has other useful information for scientists involved in dissolution testing including resource documents, recent publications, meeting minutes and current events. Please send comments or suggestions for the website to Diane Worrell at Diane.Worrell@quintiles.com or Banu Zolnik at Banu.Zolnik@uconn.edu

If you want to join this Focus Group please contact Tahseen Mirza at Tahseen.Mirza@novartis.pharma.com or go to the website listed above. 